\title{
EFFECT OF MATERIAL PROPERTIES ON THE DEFORMATION OF STEEL FRAME IN FIRE
}

\author{
By Shunsuke BABA* and Hiroshi NAGURA**
}

\begin{abstract}
In order to evalulate the effect of time-dependent material propetrities on the structural behaviour of steel frame structure in fire, a simulative study is carried out by using the experimental functions on the material properties obtained by the high temperature tensile and creep tests. The paper contains the details on the material tests. One-span one-story steel frame in fire environment is analyzed by one-dimensional FEM by assuming both small and finite deformation under the various conditions on the selection of creep property, on the selection of rate-dependent properties of elastic-limit strength, hardening parameter and modulus of elasticity, and on the selection of finite deformation assumption. A suggestion on the adequate level of exactness of the material properties is given based on the numerial results.
\end{abstract}

\section{INTRODUCTION}

Steel structures and their members in fire environment have been recently studied in an overall field by the ECCS, where numbers of research projects up to 135 subjects have been completed or are still in progress. Those projects are divided into four categories; that is, (1) general principles relating to fire, (2) properties of steel exposed to fire, (3) methods of calculating the fire behaviour of protected or unprotected steel structural elements and (4) resistance tests. Two bibliographycal reviews ${ }^{1 / 2)}$ including many papers on fire problem were reported in 1983, and the reviewers classified the papers in almost four groups such as (1) temperature at heat source, (2) material properties, (3) numerical procedures and (4) safety and design. Design recommendations have been drafted first in 1975 by the $\mathrm{CTICM}^{3}$, and the latest work was the revised recommendation by the $\operatorname{ECCS}^{4}$.

Contribution on the numerical analyses of steel structure in fire, the third group, is the fewest among the four. Structural deformation and/or resistance were studied by Barthelemy ${ }^{5)}$, Kawagoe ${ }^{6}$, Witteveen ${ }^{7}$, Kruppa $^{8)}$ and Contro ${ }^{9)}$ using the temperature-dependent material properties; however their calculations were based on the standard design formulae or ultimate design procedures. Application of FEM to the structural analysis in case of fire has been studied by few researchers as Cheng ${ }^{10)}$, Harmathy ${ }^{11)}$ and Furumura $^{12)}$ on account of its complexity.

Baba and his collaborators have been interested in the analytical, numerical and experimental works on the in-plane and out-of-plane behaviour of steel frame structures in fire environment. First a new technique using an exact form of 'warping function' calculated by the Galerkin FEM was developped ${ }^{13)}{ }^{14}$ in

* Member of JSCE, Dr. Eng., Associate Professor, Department of Geotechnical Engineering, Nagoya University (Furo-cho, Chikusa-ku, Nagoya)

** Member of JSCE, M. Eng., Engineer, Hazama-Gumi Ltd. (Kita-Aoyama 2-5-8, Minato-ku, Tokyo) 
order to analyze precisely an out-of-plane deformation of beam-column members. This procedure was applied to the out-of-plane problem of frame structure in fire ${ }^{15)}$ using the material properties and the standard-fire temperature-time curve introduced by the CTICM.

For a purpose of evaluating a difference between the approximate material properties of the CTICM and the real behaviour of structural steel material, a series of material test using the SS 41 steel specimens was executed, and modulus of elasticity, elastic limit strength, hardening property and creep strain rate were represented in the function forms by using temperature, plastic strain, strain rate and stress parameters. Applying the experimental functions directly to a one-dimensional FE analysis, fire resistance of a simple frame structure was evaluated under the small deformation assumption ${ }^{16}$.

This paper contains the details on the material tests, and mainly describes the effect of creep, rate-dependent and hardening properties on the behaviour of steel frame in fire. The authors gives a suggestion on an adequate level of exactness of the material properties used in the numerical analysis.

\section{MATERIAL TESTS OF SS 41 STEEL IN HIGH TEMPERATURE}

In the design recommendation of the CTICM and the similars, only the two material properties are identified as a temperature dependent, that is, modulus of elasticity $E$ and initial yield strength $\sigma^{E}$. However, as it is well known, structural steel in high temperature more than $\theta=400^{\circ} \mathrm{C}$ is characterized by a large amount of strain hardening and creep.

Following two experimental tests were organized by the authors, and the material properties of the SS 41 steel were formulated so as to include strain hardening, creep and rate-dependent properties :

(1) High temperature tensile test;

(2) High temperature creep test.

\section{(1) High temperature tensile test}

Static tensile test controlled under a constant strain rate was conducted by setting the temperature as $\theta$ $=\mathrm{RT}$ (room temperature) $\sim 800^{\circ} \mathrm{C}$. The rates of strain $\dot{\varepsilon}$, which were kept constant during the test, were selected as $\dot{\varepsilon}=0.1$ and $1.0 \% / \mathrm{min}$; and both results were used in evaluating the effect of strain rate on $E$ and $\sigma^{E}$. The reason why only the two kinds of strain rates were used in the test, can be found for instance in the report by Taira ${ }^{17)}$ describing a linear relation between the $0.2 \%$ proof strength of the SM $41 \mathrm{C}$ steel and $\log \dot{\varepsilon}$ for $\theta=\mathrm{RT} \sim 500^{\circ} \mathrm{C}$, and in the similar result by the ISIJ ${ }^{18)}$ describing the same relationship in the case of stainless steel for $\theta=700^{\circ} \mathrm{C} \sim$; in other
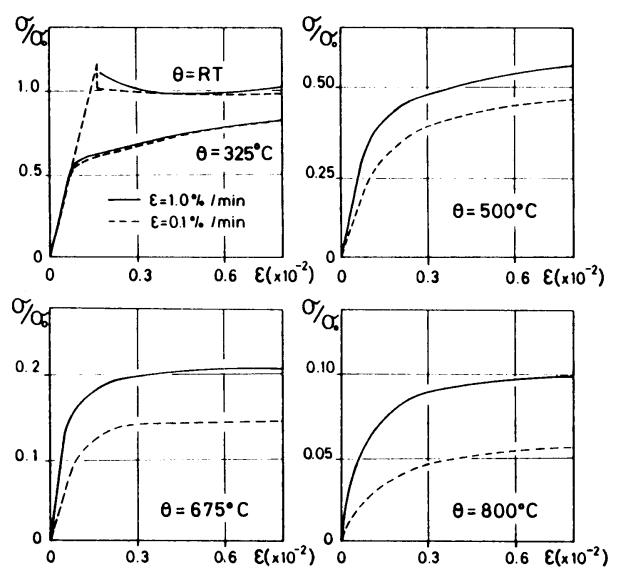

Fig. 1 Relations between Stress and Strain.
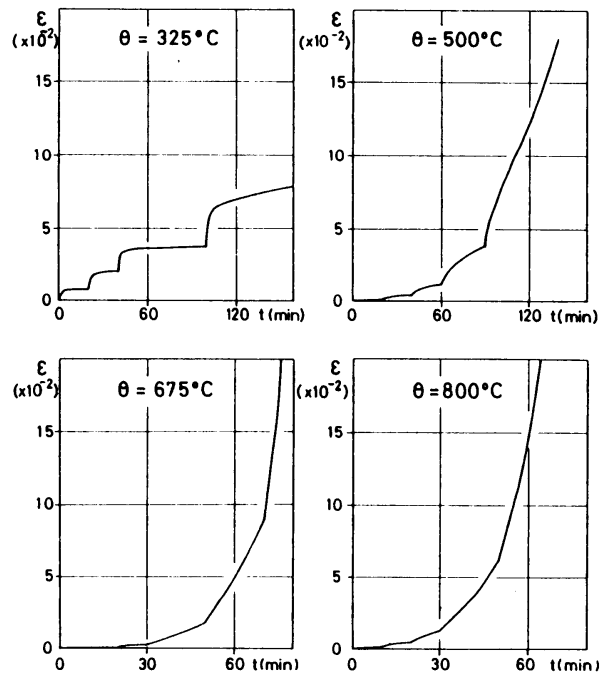

Fig. 2 Relations between Time and Strain at Different Stress Level. 
Table 1 Static Tensile Test Results.

\begin{tabular}{|c|c|c|c|c|}
\hline & \multicolumn{2}{|c|}{$\dot{\varepsilon}=1.0 \mathrm{~g} / \mathrm{min}$} & \multicolumn{2}{|c|}{$\varepsilon=0.1 \% / \mathrm{min}$} \\
\hline temperature & $\begin{array}{l}\text { elastic limit } \\
\text { strength }\end{array}$ & $\begin{array}{l}\text { modulus of } \\
\text { elasticity }\end{array}$ & $\begin{array}{l}\text { elastic limit } \\
\text { strength }\end{array}$ & $\begin{array}{l}\text { modulus of } \\
\text { elasticity }\end{array}$ \\
\hline$\theta\left({ }^{\circ} \mathrm{C}\right)$ & $\sigma_{10}^{E} / s_{0}$ & $E_{1} / E_{0}$ & $\sigma_{1.0}^{E} / \sigma_{0}$ & $E_{10} / E_{0}$ \\
\hline $\mathrm{RT}$ & 1.00 & 1.00 & 0.99 & 1.02 \\
\hline 100 & 0.97 & 0.93 & - & - \\
\hline 170 & 0.97 & 1.01 & - & - \\
\hline 225 & 0.90 & 1.07 & - & - \\
\hline 325 & 0.57 & 1.01 & 0.50 & 0.97 \\
\hline 400 & 0.42 & 0.94 & - & - \\
\hline 450 & 0.34 & 0.86 & - & - \\
\hline 500 & 0.27 & 0.76 & 0.22 & 0.39 \\
\hline 675 & 0.074 & 0.50 & 0.059 & 0.21 \\
\hline 800 & 0.038 & 0.40 & 0.018 & 0.074 \\
\hline
\end{tabular}

Table 2 Creep Test Results.

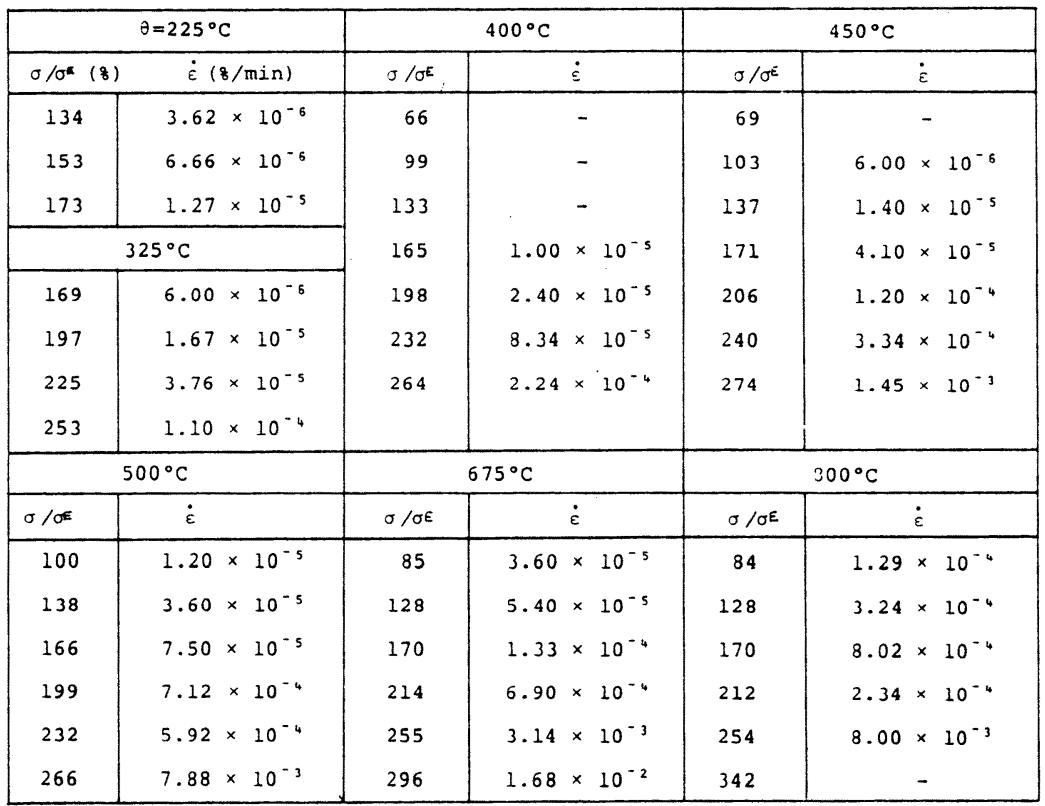

words, test results by the two kinds of different strain rate are sufficient to estimate the material properties in an arbitrary strain rate.

The test specimen was taken from a web of rolled H-type steel rod made of the SS 41 structural steel, whose section was $100 \times 100(\mathrm{~mm})$, in the accuracy of $1 / 200$. Average values of the cross-sectional area were measured, and used to correct the values of applied stress. Temperature at the specimen was controlled by an average temperature measured by each three PR-thermocouples in the accuracy of $\pm 5^{\circ} \mathrm{C}$.

Values of $E$ and $\sigma^{E}$ measured in the various temperatures are shown in Table 1. Stress-strain relationships in the case of $\theta=\mathrm{RT}, 325^{\circ} \mathrm{C}, 500^{\circ} \mathrm{C}, 675^{\circ} \mathrm{C}$ and $800^{\circ} \mathrm{C}$ are shown in Fig. 1, where strain hardening can be observed from $\theta=325^{\circ} \mathrm{C}$ and rate-dependence of the stress-strain relation appears from $\theta$ $=500^{\circ} \mathrm{C}$. In Table 1 and Fig. $1, E$ and $\sigma^{E}$ are represented by the non-dimensional forms as $E / E_{0}$ and $\sigma^{E} / \sigma_{0}$, where $E_{0}$ and $\sigma_{0}$ are modulus of elasticity and elastic-limit strength, respectively, in the case of $\theta=$ 
$\mathrm{RT}$ and $\dot{\varepsilon}=1.0 \% / \mathrm{min}$.

\section{(2) High temperature creep test}

The same specimens with the static tensile test were used. In the creep test, the specimen was loaded rapidly $(\dot{\varepsilon}=10 \% / \mathrm{min})$ until the pre-determined constant stress level and the time-strain curve was measured as shown in Fig. 2 under constant stress and constant temperature. Stress levels were selected as 3 7 steps for each temperature between $50 \%$ and $350 \%$ of $\sigma^{E}(\theta)$ in the case of $\dot{\varepsilon}=1.0 \% / \mathrm{min}$.

The test results suggest an applicability of stationary creep process. Especially in the domain of high temperature more than $500^{\circ} \mathrm{C}$, creep deformation is occupied almost by the stationary creep. Though transient creep phenomenon can be seen for a medium high temperature between $225^{\circ} \mathrm{C}$ and $325^{\circ} \mathrm{C}$, it shifts quickly to the stationary creep state. In simplifying the mathematical representation for the creep, a short-time creep which occurs during fire is assumed here as the stationary creep. Stationary creep strain rates $\dot{\varepsilon}^{c}$ calculated from the time-strain curves are given in Table 2.

\section{EXPRESSIONS OF MATERIAL PROPERTIES}

Since the purpose of this paper is to evaluate the representaion of material properties with an adequate level of accuracy, the test results of the material properties are represented in the form of series.

(1) Creep strain rate

Stress dependent property of the stationary creep strain rate $\dot{\varepsilon}^{c}$ (per minute) is represented as follows in the well-known exponential form :

$\ln \dot{\varepsilon}^{c}(\theta, \sigma)=B(\theta)\left(\sigma / \sigma_{0}\right)+A(\theta)$

Exponential expression of Eq. (1) fits well to the test results as shown in Fig. 3, where relation between non-dimensional stress $\sigma / \sigma_{0}$ and creep strain rate $\ln \dot{\varepsilon}^{c}(\theta, \sigma)$ is represented. Temperature dependent coefficients $A(\theta)$ and $B(\theta)$ are obtained as intercepts on the stress-axis and as gradients, respectively, of the straight lines in Fig. 3, and may be represented in the form of series as follows using the least mean square method:

$$
A(\theta)=\sum_{i=0}^{4} a_{i} \theta^{i}, \quad B(\theta)=\sum_{i=0}^{4} b_{i} \theta^{i}
$$

where coefficients $a_{i}$ and $b_{t}$ are indicated in Table 3 .

(2) Modification of the stress-strain relations obtained by high temperature tensile test

As will be later explained in the Section 4, the authors assume that the rate-dependent elasto-plastic property and the creep property are independent from each other. It implies the yield state is defined by excluding the effect of creep strain. Since creep strain component $\varepsilon^{c}$ is included in the total strain $\varepsilon$ measured by the static tensile test, the $\sigma-\varepsilon$ relations used in the yield function should be modified so as to neglect the corresponding amount of $\varepsilon^{c}$ from the total strain $\varepsilon$.

Creep strain $\varepsilon^{c}\left(t^{*}\right)$, which will be observed after $t^{*}$ minutes from a start of loading in the static tensile test, is expressed as follows by using Eq. (1) :

$$
\begin{aligned}
\varepsilon^{c}\left(t^{*}\right) & =\int_{0}^{t^{*}} \dot{\varepsilon}^{c} d t \\
& =\int_{0}^{t} \exp [A(\theta)] \exp \left[B(\theta)\left(\sigma / \sigma_{0}\right)\right] d t
\end{aligned}
$$

The $\sigma-\varepsilon$ relations obtained by a constant strainrate test ia also recognized as the $\sigma-t^{*}$ relations, therefore the integration by time in Eq. (2) can be used directly in the modification of the $\sigma-\varepsilon$ $\left(\sigma-t^{*}\right)$ curves.

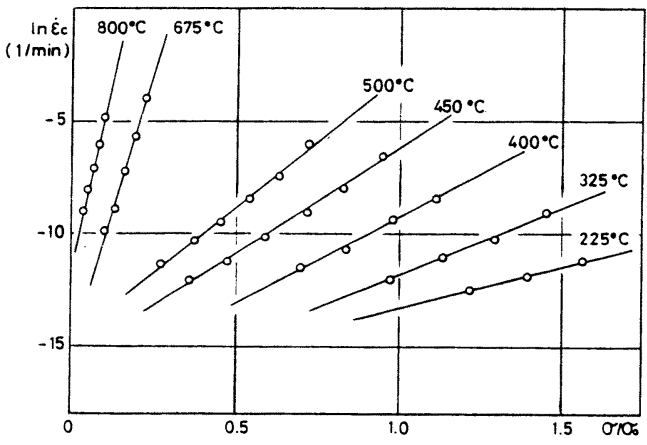

Fig. 3 Relations between Stress and Stationary Creep Strain Rate. 
Table 3 Coefficients of Parameters $A \sim H$.

\begin{tabular}{|c|c|c|c|}
\hline \multicolumn{2}{|r|}{ creep strain rate } & \multicolumn{2}{|r|}{ strain hardening } \\
\hline$a_{0}$ & $0.2243434 \times 10^{2}$ & $f_{0}$ & $0.7022064 \times 10^{\circ}$ \\
\hline$a_{1}$ & $-0.3856286 \times 10^{\circ}$ & $f_{1}$ & $0.1002840 \times 10^{1}$ \\
\hline$a_{2}$ & $0.1298603 \times 10^{-2}$ & $\mathbf{f}_{2}$ & $0.9999927 \times 10^{\circ}$ \\
\hline d: & $-0.1799622 \times 10^{-5}$ & $\mathrm{f}_{3}$ & $0.1000000 \times 101$ \\
\hline a, & $0.8917637 \times 10^{-9}$ & f 4 & $0.1000000 \times 10^{1}$ \\
\hline$b_{0}$ & $-0.2855091 \times 10^{4}$ & 90 & $-0.1330370 \times 10^{\circ}$ \\
\hline$b_{1}$ & $0.3162711 \times 10^{2}$ & 91 & $0.1287936 \times 10^{-2}$ \\
\hline$b_{2}$ & $-0.1187413 \times 10^{\circ}$ & 9. & $-0.4744697 \times 10^{-9}$ \\
\hline bs & $0.1849584 \times 10^{-3}$ & $y_{3}$ & $0.8867404 \times 10^{\circ}$ \\
\hline \multirow[t]{3}{*}{ b、 } & $-0.9584928 \times 10^{-7}$ & 94 & $-0.9062770 \times 10^{-11}$ \\
\hline & & ys & $0.4885456 \times 10^{-12}$ \\
\hline & & $y_{6}$ & $-0.1102996 \times 10^{-11}$ \\
\hline \multicolumn{2}{|c|}{ elastic-limit strength } & \multicolumn{2}{|c|}{ modulus of elasticity } \\
\hline$c_{0}$ & $0.3586533 \times 10^{-1}$ & ev & $0.5220441 \times 10^{0}$ \\
\hline$c_{1}$ & $-0.7839153 \times 10^{-6}$ & $e_{1}$ & $-0.1688540 \times 10^{-2}$ \\
\hline $\mathrm{c}_{2}$ & $0.1572770 \times 10^{-5}$ & $e_{2}$ & $0.1825000 \times 10^{-4}$ \\
\hline$c_{1}$ & $-0.1106798 \times 10^{-1}$ & e, & $-0.6796975 \times 10^{-7}$ \\
\hline c、 & $0.3183328 \times 10^{-10}$ & es & $0.1085616 \times 10^{-9}$ \\
\hline cs & $-0.4520540 \times 10.13$ & es & $-0.7995861 \times 10^{-13}$ \\
\hline$c$ & $0.3160878 \times 10^{-16}$ & e。 & $0.2244941 \times 10^{-16}$ \\
\hline$\therefore$ & $-0.8698310 \times 10^{-20}$ & $h_{0}$ & $-0.6586500 \times 10^{-1}$ \\
\hline$d_{0}$ & $0.1017796 \times 10^{\prime}$ & $h_{1}$ & $0.4242302 \times 10^{-3}$ \\
\hline$d_{\mathfrak{l}}$ & $-0.5969708 \times 10^{-4}$ & 1.2 & $0.8878937 \times 10^{-6}$ \\
\hline d, & $0.8498541 \times 10^{\circ}$ & & \\
\hline
\end{tabular}

All results in the static tensile test are modified by subtracting $\varepsilon^{c}$ calculated by Eq. (2) from the $\sigma-\varepsilon$ relations. Fig. 4 shows an example of the modification, where the relations between stress $\sigma / \sigma_{0}$ and (1) measured total strain $\varepsilon$, (2) corresponding creep strain $\varepsilon^{c}$ and (3) modified strain $\left(=\varepsilon-\varepsilon^{c}\right)$ are indicated in the case of $\theta=$ $800^{\circ} \mathrm{C}$ and $\dot{\varepsilon}=0.1 \% / \mathrm{min}$. Amount of the

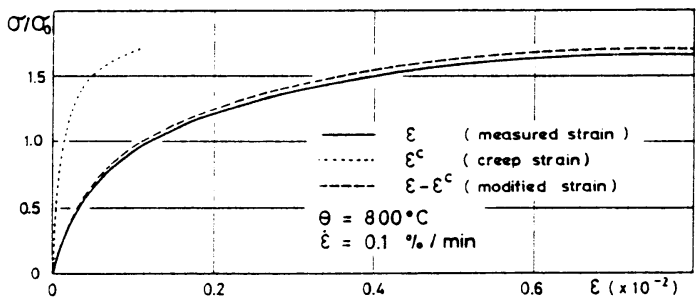

Fig. 4 Modification of the Stress-Strain Relation. modification, in this case, reaches up to $13.5 \%$ of the measured strain.

\section{(3) Elastic-limit strength and strain hardening}

Yield parameter $\sigma^{Y}$ is defined here as a combination of the elastic-limit strength $\sigma^{E}$ and the subsequent stress increase $\sigma^{H}$ due to strain hardening. Yield parameter $\sigma^{r}$ is used in determing the size of yield surface, and is a function of temperature $\theta$, plastic strain $\varepsilon^{\rho}$ and strain rate $\dot{\varepsilon}$; and it may be represented simply as separate form as follows:

$$
\sigma^{\gamma}\left(\theta, \varepsilon^{p}, \dot{\varepsilon}\right)=\sigma_{1.0}^{E}(\theta) P(\theta, \dot{\varepsilon})+\sigma^{H}\left(\theta, \varepsilon^{p}\right)
$$

in which $\sigma_{1.0}^{E}(\theta)$ is the elastic-limit strength in the case of $\dot{\varepsilon}=1.0 \% / \mathrm{min}$, and the variation of $\sigma^{E}$ due to strain rate $\dot{\varepsilon}$ is described by parameter $P(\theta, \dot{\varepsilon})$. Stress increase $\sigma^{H}\left(\theta, \varepsilon^{p}\right)$ due to strain hardening is assumed as independent with strain rate $\dot{\varepsilon}$, because the influence of strain rate on the hardening part is small, as indicated later in Fig. 5.

Elastic-limit strength for $\dot{\varepsilon}=1.0 \% / \mathrm{min}$ may be expressed by an interpolation function for the temperature $\theta$ as follows:

$$
\sigma_{1.0}^{E}(\theta)=\sigma_{0} C(\theta), \quad C(\theta)=\sum_{i=0}^{7} c_{i} \theta^{\imath}
$$


in which coefficients $c_{i}$ are indicated in Table 3.

Elastic-limit strength $\sigma^{E}(\theta, \dot{\varepsilon})$ for an arbitrary strain rate $\dot{\varepsilon}$ is estimated as follows using both $\sigma_{1.0}^{E}(\theta)$ and $\sigma_{0.1}^{E}(\theta)$, where the latter is an elastic-limit strength in the case of $\dot{\varepsilon}=0.1 \%$ $/ \mathrm{min}$ :

$$
\begin{aligned}
\sigma^{E}(\theta, \quad \dot{\varepsilon}) & =\sigma_{1.0}^{E}(\theta)\left[1+\left(1-\sigma_{0.1}^{E}(\theta) / \sigma_{1.0}^{E}(\theta)\right) \log \dot{\varepsilon}\right] \\
& =\sigma_{0} C(\theta)[1+D(\theta) \log \dot{\varepsilon}]=\sigma_{0} C(\theta) P(\theta, \dot{\varepsilon})
\end{aligned}
$$

Range of strain rate, for which Eq. (4) is applicable, is regarded as $\dot{\varepsilon}=0.05 \sim 5 \%$ $/ \mathrm{min}$; that is $\sigma^{E}(\theta, \dot{\varepsilon}=0.05 \%)$ and $\sigma^{E}(\theta, \dot{\varepsilon}=$ $5 \%$ ) become a lower and an upper bound of possible value for the elastic-limit strength. Parameter $D(\theta)$ may be represented as

$$
D(\theta)=\sum_{i=0}^{2} d_{i} \theta^{i}
$$

where coefficients $d_{i}$ are indicated in Table 3.

Shape of the strain hardening part represented by $\sigma^{H}\left(\theta, \varepsilon^{D}\right)$ is assumed as follows based on the test results :

$$
\sigma^{H}\left(\theta, \varepsilon^{p}\right)=\sigma_{0}\left[F(\theta)+G(\theta) \ln \varepsilon^{p}\right]
$$

Relation between stress $\sigma / \sigma_{0}$ and plastic strain $\ln \varepsilon^{\rho}$ is represented in Fig. 5, and it verifies an almost linear relation between $\sigma / \sigma_{0}$ and $\ln \varepsilon^{p}$ as assumed in Eq. ( 5 ). In Fig. 5, the linear relations both for $\dot{\varepsilon}=$ 1.0 and $0.1 \% / \mathrm{min}$ are shown, and the figure says that the influence of the strain rate on the hardening property is rather small and can be neglected in the first approximation. Parameters $F(\theta)$ and $G(\theta)$ may be represented by the following expressions [ $f_{i}$ and $g_{t}$ are expressed in Table 3]:

$$
F(\theta)=\sum_{i=0}^{4} f_{i} \theta^{i}, \quad G(\theta)=\sum_{i=0}^{6} g_{i} \theta^{i}
$$

Combining Eqs. (3) $-(5)$, yield parameter $\sigma^{\gamma}\left(\theta, \varepsilon^{p}, \dot{\varepsilon}\right)$ can be finally expressed as follows using the known functions $C(\theta), D(\theta), F(\theta), G(\theta)$ and variables $\varepsilon^{p}$ and $\dot{\varepsilon}$ :

$$
\sigma^{\mathrm{Y}}\left(\theta, \quad \varepsilon^{p}, \dot{\varepsilon}\right)=\sigma_{0}\left\{C(\theta)[1+D(\theta) \log \dot{\varepsilon}]+F(\theta)+G(\theta) \ln \varepsilon^{p}\right\}
$$

(4) Modulus of elasticity

In the same way as for the elastic-limit strength, modulus of elasticity $E(\theta, \dot{\varepsilon})$ for an arbitrary strain rate $\dot{\varepsilon}$ is represented as follows using $E_{1.0}(\theta)[=E(\theta, \dot{\varepsilon}=1.0 \% / \mathrm{min})]$ and $E_{0.1}(\theta)[=E(\theta, \dot{\varepsilon}=0.1 \%$ $/ \min )]$ :

$$
\begin{aligned}
E(\theta, \dot{\varepsilon}) & =E_{1.0}(\theta)\left[1+\left(1-E_{0.1}(\theta) / E_{1.0}(\theta)\right) \log \dot{\varepsilon}\right] \\
& =E_{0} E(\theta)[1+H(\theta) \log \dot{\varepsilon}] \ldots \ldots \ldots \ldots \ldots \ldots \ldots \ldots \ldots
\end{aligned}
$$

where

$$
E(\theta)=\sum_{i=0}^{6} e_{i} \theta^{i}, \quad H(\theta)=\sum_{i=0}^{2} h_{i} \theta^{i}
$$

whose coefficients $e_{i}$ and $h_{i}$ are given in Table 3 .

\section{CONSTITUTIVE LAW AND FEM}

\section{(1) Constitutive law}

Constitutive laws proposed for a viscoelastic-plastic material is almost divided into two groups, that is, uncoupled and coupled methods. In the former one, viscoelastic-plastic behaviour is represented by an uncoupled - independent-superposition of viscous (creep) and elasto-plastic behaviour; it implies the yield of material is only concerned with the plasticity and has no relation with the creep. The method 
developped by Landau ${ }^{19)}$, Yagawa ${ }^{20)}$, et al. is simple and rather classical, and is said to be applied widely in a sufficiently good accuracy to the elasto-plastic problem with creep deformation.

In the latter (coupled) one, basic idea was originated from a visco-plastic model proposed by Perzyna ${ }^{21)}$ and $\mathrm{Naghdi}^{22}$, and has recently been used in analyzing both an extremely rapid process and a very slow process. The method shows a high applicability to a rate-dependent elasto-plastic problem as seen in a high-speed shock response of metal, and also to a long-term creep problem as observed in a deformation of rock.

In a domain of high temperature more than $\theta=400^{\circ} \mathrm{C}, E$ and $\sigma^{E}$ show a strong dependence on the strain rate $\dot{\varepsilon}$, and besides a large amount of creep strain $\varepsilon^{c}$ occurs. However, the combined effect of $\dot{\varepsilon}$ and $\varepsilon^{c}$ is difficult to be represented by using the latter (coupled) method. The authors take the former (uncoupled) method, and divide the time-dependent behaviour caused by $\dot{\varepsilon}$ and $\varepsilon^{c}$ into two independent parts, that is, (1) rate-dependent thermo-elasto-plastic component with strain hardening and (2) stationary creep component. The yield of material is, in this case, related only to the component (1).

( 2 ) Stress-strain relation

Following tensor relation is assumed among stress increment $d g$, elastic stiffness constant $\underset{\sim}{C}$, elastic strain $\underset{\underset{\varepsilon}{e}}{e}$ and their increments $d \underset{\sim}{C}$ and $d \varepsilon^{e}$ :

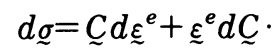

where the second term implies an incremental stress occurred by the variation of $\underset{\sim}{C}$ due to incremental temperature $d \theta$. Since the total strain increment tensor $d \xi$ can be represented as an independent tensor summation of $d \xi^{e}$, plastic stain increment $d \varepsilon^{p}$, thermal strain increment $d \varepsilon^{\theta}$ and creep strain increment $d \varepsilon^{c}$ as

$$
d \underline{\varepsilon}=d \underline{\varepsilon}^{e}+d \underline{\varepsilon}^{p}+d \underline{\varepsilon}^{\theta}+d \underline{\varepsilon}^{c}
$$

Eq. ( 8 ) is represented as follows using the associated flow rule :

$$
\begin{aligned}
& d \sigma=\underset{\sigma}{C}\left(d \underline{\varepsilon}-d \varepsilon^{p}-d \varepsilon^{\theta}-d \varepsilon^{c}\right)+\underline{\varepsilon}^{e} d \underline{G} \\
& =C\left(d \underline{\varepsilon}-d \underline{\varepsilon}^{\theta}-d \underline{\varepsilon}^{c}\right)-\Lambda_{p} \underline{\underline{S}}+\underline{\varepsilon}^{e} d \underline{C}
\end{aligned}
$$

in which $\underline{s}$ is deviatoric stress tensor. Positive scalar function $\Lambda_{p}$ is determined here by assuming the von Mises yield criterion and an isotropic strain hardening. Yield function $\mathrm{f}$ is written as

$$
f=[(3 / 2)(\underline{s}: \underline{s})]^{\frac{1}{2}}-\sigma^{\mathrm{Y}}\left(\theta, \bar{\varepsilon}^{p}, \dot{\bar{\varepsilon}}\right)
$$

where bar over $\varepsilon$ implies an equivalent strain, and $\sigma^{Y}$ is substituted by Eq. (6). Using the Prager's consistency condition $d f=0$ to an equation for $f, \Lambda_{p}$ in Eq. (10) is determined as follows:

$$
\Lambda_{p}=\left(3 / 2 \sigma^{\gamma} H^{\prime}\right)\left\{\left(3 / 2 \sigma^{\gamma}\right)[(g: d g)-(1 / 3)(\operatorname{tr} g)(\operatorname{tr} d \sigma)]-\left(\partial \sigma^{\gamma} / \partial \theta\right) d \theta-\left(\partial \sigma^{\gamma} / \partial \dot{\bar{\varepsilon}}\right) d \dot{\bar{\varepsilon}}\right\}
$$
where $H^{\prime}\left(\theta, \bar{\varepsilon}^{p}\right)=\partial \sigma^{H}\left(\theta, \bar{\varepsilon}^{p}\right) / \partial \bar{\varepsilon}^{p}$, and $\sigma^{H}$ is given by Eq. $(5)$.

Strain increments $d \varepsilon^{\theta}$ and $d \varepsilon^{c}$ in Eq. (10) are calculated as

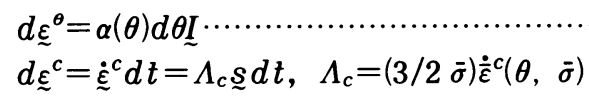

in which $\underset{I}{I}$ is a unit tensor, $\bar{\sigma}$ is an equivalent stress and $\alpha(\theta)$ is a coefficient of thermal expansion defined as $^{23)} \alpha(\theta)=1.3 \times 10^{-8} \theta+1.1 \times 10^{-5}$. Eq. (13) is obtained by assuming that $d \varepsilon^{c}$ is represented as a product of creep strain rate $\dot{\varepsilon}^{c}$ and time increment $d t^{24)}$, and that $\dot{\xi}^{c}$ is described by an equivalent creep strain rate $\dot{\bar{\varepsilon}}^{c}$ given by Eq. (1).

Eqs. (10) - (13) are represented in the simple forms as follows in the case of one-dimensional problem :

$$
\begin{aligned}
& d \sigma_{x}=\frac{E H^{\prime}}{E+H^{\prime}}\left(d \varepsilon_{x}-d \varepsilon_{x}^{\theta}-d \varepsilon_{x}^{c}\right)+\left(\operatorname{sign} \sigma_{x}\right) \frac{E}{E+H^{\prime}}\left(\frac{\partial \sigma^{\gamma}}{\partial \theta} d \theta+\frac{\partial \sigma^{\gamma}}{\partial \dot{\bar{\varepsilon}}} d \dot{\bar{\varepsilon}}\right)+\frac{H^{\prime}}{E+H^{\prime}} \varepsilon_{x}^{e} d E \\
& d \varepsilon_{x}^{\theta}=\alpha(\theta) d \theta \\
& d \varepsilon_{x}^{c}=\dot{\varepsilon}^{c} d t
\end{aligned}
$$

in which $E, H^{\prime}, \sigma^{\gamma}$ and $\dot{\varepsilon}^{c}$ are functions defined by Eqs. (7), (5), (6) and (1), respectively, and $\left(\operatorname{sign} \sigma_{x}\right)=\left|\sigma_{x}\right| / \sigma_{x}$.

The first term of right hand side of Eq. (14) implies a stress variation due to an instantanous cramp of 
thermal and creep strains caused by a temperature variation, or otherwise an incremental stress due to an applied load. The second term inplies a stress variation due to a reduction(or extension) of yield surface caused by a temperature variation. The third term implies a stress variation due to a decrease (or increase) of modulus of elasticity.

\section{(3) FE analysis - Deformation}

Two-dimensional steel frame structure with bi-axially symmetrical $\mathrm{H}$-section is analyzed using the one-dimensional FEM under the assumptions as (1) small deformation and finite deformation, (2) in-plane deformation and (3) Bernoulli-Euler's assumption.

Virtual work described only by $\sigma_{x}$ and $\varepsilon_{x}$ is represented in an incremental form based on the Total Lagrangean Formulation. Three-nodes seven-degrees-of-freedom beam element is used, and each element is divided into five Gaussian points in the axial direction. Cross-section of the element, at each Gaussian point, is divided into 37 integration points. Detailed explanation on the $\mathrm{FE}$ formulation can be seen in the Ref. (14).

\section{(4) FE analysis - Temperature}

Time-temperature relation at the heat source is substiuted by the standard fire time-temperature curve ${ }^{3)}$. The heat transfer from the heat source to the structural member is assumed to be carried by a convection and radiation ${ }^{23}$. Thermal boundary condition is shown in Fig. 7, where heat transfer due to radiation is done only along the lower surface of flange although heat transfer due to convection occurs at all surface of the section. Since the heat transfer is carried only by a difference of temperature between the heat source and the surface of member, temperature distribution at the structural member can be assumed to have the same pattern each other as long as it has the same cross-section.

Heat transfer analysis is conducted by the Galerkin FEM using the four-nodes four-degrees-of-freedom rectangular element. Detailed formulation is seen in the Ref. (15). Coefficient of heat conductivity and specific heat of steel follow the CTICM's recommendation ${ }^{3)}$. Integration by time is carried by the Crank-Nicholson method, and the temperature distribution of the section is calculated at intervals of $5 \sim 10$ seconds. Typical examples of temperature distribution at every five minutes after a break of fire are shown in Fig. 6.

\section{SIMULATIVE STUDY ON THE SELECTION OF MATERIAL PROPERTIES}

\section{(1) Numerical example}

One-span one-story frame structure as shown in Fig. 7 is analyzed as an example. Since the slenderness ratios of the members are low, an effect of sway deformation on the total deformation of the frame is neglected.

In order to obtain an adequate number of element division, the frame is cut into different numbers of element and analyzed under the same load and the same uniform temperature $\left(\theta=600^{\circ} \mathrm{C}\right)$. Fig. 8 shows the vertical displacement $\delta / \delta_{n=32}$ at the center of frame in case that the structure is discretized into $n=8,16$, 24 and 32 elements, where $\delta_{n=32}$ implies the displacement in the case of $n=32$. It is concluded the number of element should be more than 16 , and is preferable to be more than 24 .

\section{(2) Effect of strain rate}

Strain rate is contained in the functions of $E(\theta, \dot{\varepsilon})$ and $\sigma^{\gamma}\left(\theta, \varepsilon^{p}, \dot{\varepsilon}\right)$ as expressed in the Section 3. However an existence of the strain rate in those functions brings an unstable convergence of numerical calculation as explained in the followings.

Strain rate cannot be known until the strain itself is obtained because strain rate at the $n$-th step $\dot{\varepsilon}_{n}$ is determined as $\dot{\varepsilon}_{n}=\left(\varepsilon_{n}-\varepsilon_{n-1}\right) / d t$, in which $\varepsilon_{n}$ and $\varepsilon_{n-1}$ are strains at the $n$-th and $(n-1)$ th steps, respectively. On the contrary, $\dot{\varepsilon}_{n}$ is used in the calculation of $\varepsilon_{n}$ because the rate-dependent values $E$ and $\sigma^{\gamma}$ are functions of $\dot{\varepsilon}_{n}$. It implies an assumed value of $\dot{\varepsilon}_{n}^{*}$ should be used instead of the unknown $\dot{\varepsilon}_{n}$.

Strain rate $\dot{\varepsilon}_{n}^{*}$ is assumed in the following three ways: 
(1) To make an simulative calculation until $\dot{\varepsilon}_{n}^{*}=\dot{\varepsilon}_{n}$, that is, until the assumed and resulted strain rates $\left(\dot{\varepsilon}_{n}^{*}\right.$ and $\left.\dot{\varepsilon}_{n}\right)$ become similar each other;

(2) To assume as $\dot{\varepsilon}_{n}^{*}=\dot{\varepsilon}_{n-1}$, that is, to substitute $\dot{\varepsilon}_{n}^{*}$ by the known strain rate of the old step $\dot{\varepsilon}_{n-1}$;

(3) To cramp $\dot{\varepsilon}_{n}^{*}$ as $\dot{\varepsilon}_{n}^{*}=\dot{\varepsilon}=$ constant, that is, neglect the rate-dependent property of $E$ and $\sigma^{Y}$.

The frame structure with uniform load $q_{0}$ [Fig. 7], whose magnitude correponds to 60 percent of the initial yield load, is calculated using three kinds of $\dot{\varepsilon}_{n}^{*}$ based on the small deformation assumption. Results are shown in Fig. 9, where the relations between time $t$ (minutes) from a break of fire and vertical deformation $\delta$ at the center of frame are indicated. Vertical deformation is represented in the non-dimensional form using $\delta_{0}$ (value of $\delta$ at the time $t=0$ ).

Following conclusions are obtained from Fig. 9 :

[a ] Calculation for the case (2), where $\dot{\varepsilon}_{n}^{*}=\dot{\varepsilon}_{n-1}$, gives an unstable result. The reason of bad convergence is considered as follows. When $\dot{\varepsilon}_{n}^{*}$ is once estimated to be smaller than its true value, it produces a large plastic strains because the yield surface reduces, and therefore $\dot{\varepsilon}_{n+1}$ is evaluated as the larger value than its true one. On the contrary, when $\dot{\varepsilon}_{n}^{*}$ is estimated to be larger than its true value, it produces few plastic strains (sometimes no plastic strain) because the yield surface extends, and therefore $\dot{\varepsilon}_{n+1}$ is estimated as the smaller value than its true one;

[b] Calculation for the cases (1) and (3) gives the similar result each other in Fig. 9 [also in the stress distributions, though they are not indicated here]. The case $(1)$ is almost equivalent to the case ( 3 ), where $\dot{\varepsilon}=0.1 \% / \mathrm{min}$, at the first five minutes from a break of fire. But after the five minutes, the case (1) transfers to the case (3) where $\dot{\varepsilon}=1.0 \% / \mathrm{min}$. It can be concluded the use of case ( 3 ), where $\dot{\varepsilon}=1.0 \% / \mathrm{min}$, is recommendable instead of the case (1), because it will save much calculation time as well as it gives the similar result.

\section{(3) Effect of creep and strain hardening}

It has been concluded in the Section 5.2 that the rate-dependent property for $E$ and $\sigma^{Y}$ can be neglected without decreasing their accuracy. Section 5.3 treats the creep strain and the strain hardening.

Following four cases are calculated :

(1) To fix the strain rate as $\dot{\varepsilon}=1.0 \% / \mathrm{min}$. It implies parameters $D(\theta)$ and $H(\theta)$ are zero;

(2) To follow the case (1), and further to set the creep strain $\varepsilon^{c}$ always as $\varepsilon^{c}=0$. It implies parameters $A(\theta), B(\theta), D(\theta)$ and $H(\theta)$ are zero;

(3) To follow the case (2), and further to assume the elasto-perfectly plastic material. It implies parameters $A(\theta), B(\theta), D(\theta), F(\theta), G(\theta)$ and $H(\theta)$ are zero;

(4) To use the CTICM's material properties for $E$ and $\sigma^{E}$, that is, to assume the elastio-perfect plastic material similar with the case (3), where only one difference is in the shape of interpolation functions for $E$ and $\sigma^{E}$.

The same structure as the Section 5.2 is analyzed under the four kinds of situation as (1), (2), (3) and (4). Numerical results are shown in Fig. 10 in the same way as Fig.9.

Following conclusions are obtained from Fig. 10 :

[a] Calculation for the case (2) gives a little bit larger deformation than the case (1). Constrained

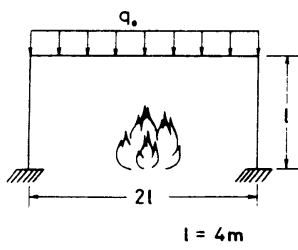

Fig. 6 Frame Structure in Fire.
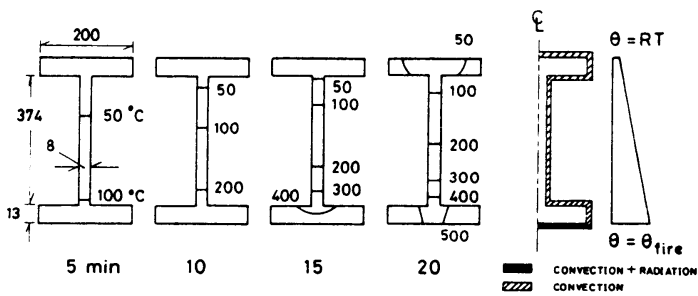

Fig. 7 Thermal Boundary Condition and Temperature Distributions. 


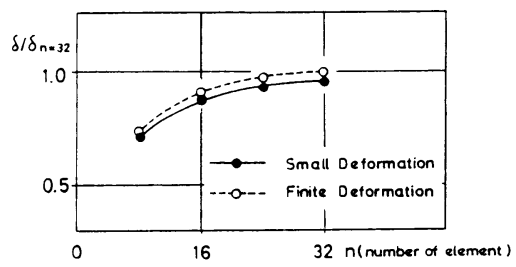

Fig. 8 Relation between Accuracy and Number of Element.

structure in fire is characterized by a stress reduction due to relaxation, which serves to decrease a total deformation. And therefore, if creep deformation is ignored, the deformation is possible to increase :

[b] Calculation for the case (3) gives an extremely larger deformation than the case $(1)$. It implies the effect of strain hardening is serious;

[c] Calculation for the case (4) gives an intermediate result between cases (1) and (3). It implies the yield strength specified by the CTICM is determined as being larger than the elastic-limit strength in order to evaluate the hardening part.

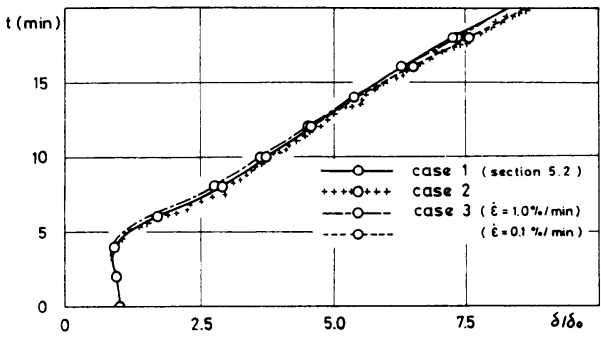

Fig. 9 Comparison of Time-Deformation Curves (Section 5.2)

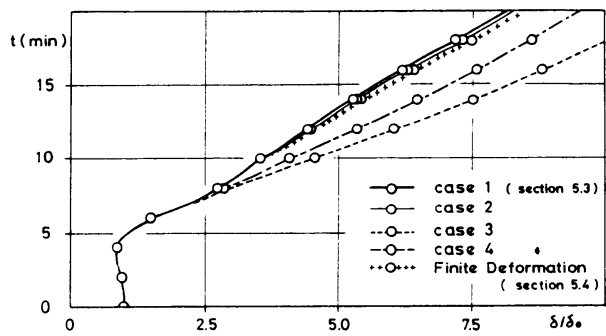

Fig. 10 Comparison of Time-Deformation Curves (Sections 5. 3,5.4).

\section{(4) Effect of finite deformation}

The same structure as in one in the Section 5.2 is also analyzed in the case of $\dot{\varepsilon}=1.0 \% / \mathrm{min}$ based on the finite deformation assumption. Result is also shown in Fig. 10, and can be compared with the result by the small deformation assumption. Difference between the finite and the small deformation is significant, therefore the use of the finite deformation assumption is preferable.

\section{CONCLUSION}

Two kinds of the high temperature material test, that is, tensile and creep tests were executed, and the three kinds of material property such as the modulus of elasticity $E(\theta, \dot{\varepsilon})$, yield parameter $\sigma^{\gamma}\left(\theta, \varepsilon^{p}, \dot{\varepsilon}\right)$ and stationary creep rate $\dot{\varepsilon}^{c}(\theta, \sigma)$ were represented in the function forms using the temperature $\theta$, plastic strain $\varepsilon^{p}$, strain rate $\dot{\varepsilon}$ and stress $\sigma$ as parameters.

Constitutive law has been formulated by assuming a thermo-viscoelastic-plastic behaviour as an independent sum of the rate-dependent thermo-elasto-plastic component and the stationary creep component, and by assuming a yield of material to be dependent only on the former component.

One-span one-story steel frame structure with uniform load in fire environment has been analyzed by the one-dimensional FEM, where the temperature distribution of the cross-section of the member is calculated by the two-dimensional Galerkin FEM in every five or ten seconds from a break of fire. The relations between time $t$ and deflection $\delta$ at the center of the frame have been calculated in the various cases in order to know the effect of rate-dependent properties of $E$ and $\sigma^{E}$, effect of creep and strain hardening, and the effect of finite deformation assumption on the structural behaviour of steel frame in fire

The following conclusions have been obtained :

[a] Rate-dependent properties on $E$ and $\sigma^{E}$ can be neglected, and the constant strain rate corresponding to $\dot{\varepsilon}=1.0 \% / \mathrm{min}$ is recommended;

[ b ] Creep has a small effect on the total deformation, though it may have a large effect on the stress state. Plastic deformation becomes more and more decreased as creep deformation goes on increasing, 
because the internal stress reduces due to relaxation;

[c ] Strain hardening should not be neglected in the fire problem. The hardening property at the high temperature gives the larger effect on the structural analysis than it gives in the case of room temperature;

[d] Finite deformation assumption is preferable in the fire problem, because structure in fire generates the larger deformation than it can behave on the normal state.

The resistance and the safety of the frame structure during and after fire will be discussed in our next report. The tests described here were executed mainly by T. Yamamoto (Sumitomo Heavy Industry Ltd. ) at the Central Technical Research Laboratory of Chubu Electric Power Co. Inc.. Computations were carried out on FACOM M-230 in the Nagoya University Computer Center.

\section{REFERENCES}

1) Law, M. : A basis for the Design of Fire Protection of Building Structures, Structural Eng., 61 A. 1, pp. 25 33, 1983.

2) Brozzetti, J., Law, M., Pettersson, O. and Witteveen, J. : Safety Concept and Design for Fire Resistance of Steel Structures, IABSE Periodica, 1, pp. 1 27, 1983.

3) CTICM (Centre Technique Industriel de la Construction Métallique) : Prévision par le Calcul du Comportement au Feu des Structures en Acier, Project de Recommendations, 76 p., 1975.

4) ECCS (European Convention for Constructional Steelwork) : European Recommendations for the Fire Safety of Steel Structures, Elsevier, 106 p., 1982.

5) Barthelemy, B. : Heating Calculation of Structural Steel Members, Proc. ASCE, 102 (ST 8), pp. 1549 1558, 1976.

6) Kawagoe, K. and Saito, H. : Thermal Effects of Fires in Buildings, IABSE, 10th Congress, Tokyo, pp. 109 123, 1976.

7) Witteveen, J., Twilt, L. and Bijlaard, F. S. K. : Theoretical and Experimental Analysis of Steel Structures at Elevated Temperatures, IABSE, 10th Congress, Tokyo, pp. 183 188, 1976.

8) Kruppa, J. : Collapse Temperature of Steel Structures, Proc. ASCE, 105 (ST 9), pp. 1769 1788, 1979.

9) Contro, R. and Giacomini, S. : A Method for Analyzing Frame Structures Exposed to Fire, Costruzioni Metalliche, 2, pp. 2 $8,1981$.

10) Cheng, W. C. and Mak, C. K. : Computer Analysis of Steel Frame in Fire, Proc. ASCE, 101 (ST 4 ), pp. 855 867, 1975.

11) Harmathy, T. Z. : Creep Deflection of Metal Beams in Transient Heating Processes- with Particular Reference to Fire, Can. J. Civil. Eng., 3, p. 219, 1976.

12) Furumura, F. and Shinohara, Y. : Inelastic Behavior of Protected Steel Beams and Frames in Fire, Rep. Res. Lab. of Engng. Mater., Tokyo, 3, pp. 1 14, 1978.

13) Baba, S. and Kajita, T. : Plastic Analysis of Torsion of a Prismatic Beam, Int. J. Numer. Meth. Engng., 18, pp. 927 944, 1982.

14) Baba, S. and Yamamoto, T. : On the Evaluation of Warping Function of Beam under Combined Torsion (in Japanese), Proc. JSCE, 334, pp. 35 42, 1983.

15) Baba, S. and Kajita, T. : Out-of-Plane Deformation of Steel Frame in Fire, Proc. 1st. Int. Conf. on Computing in Civil Engng., New York, pp. 343 358, 1981.

16) Baba, S. and Yamamoto, T. : On the Strength of Steel Frame Structures in Fire, Proc. IABSE Symp. on Strengthening of BuildingStructures Diagnosis and Therapy, Venezia, pp. 243 250, 1983.

17) Taira, S. : The 2nd. Report of Comparative High Temperature Tensile Testing of Steels (in Japanese), J. ISIJ, 55. 10, p. 932, 1969.

18) ISIJ (Iron and Steel Institute of Japan)-Creep Committee : The 6th Report of Comparative High Temperature Tensile Testing of Steels (in Japanese), ISIJ, 1977.

19) Landau, H. G., Weiner, J.H. and Zwicky Jr., E.E. : Thermal Stress in a Viscoelastic-Plastic Plate with Temperature Dependent Yield Stress, J. Appl. Mech., 27, pp. 297 302, 1960.

20) Yagawa, M. and Miyazaki. N. : Present Situation of Creep Analysis by FEM (in Japanese), J. JSME, 79, pp. 532 538, 1976.

21) Perzyna, P. : The Constitutive Equations for Rate Sensitive Plastic Materials, Tech. Rep. 76, Brown Univ., 1962.

22) Naghdi, P. M. and Murch, S. A. : On the Mechanical Behavior of Viscoelastic/Plastic Solids, J. Appl. Mech., 30, pp. 321 328, 1963.

23) Dotreppe, J.C. : Prévision par le Calcul du Comportement à l'Incendie des Structures Simples, Rep., Service Ponts et Charpentes, Univ. de Liège, 124 p., 1976.

24) Zienkiewicz, O.C. and Cormeau, I. C. : Visco-Plasticity/Plasticity and Creep in Elastic Solids, Int. J. Numer. Meth. Engng. 8, pp. 821 845, 1974.

25) Mroz, Z. : Mathematical Models of Inelastic Material Behaviour, Solid Mech. Div., Univ. of Waterloo, 160 p., 1973.

(Received May 4 1984) 